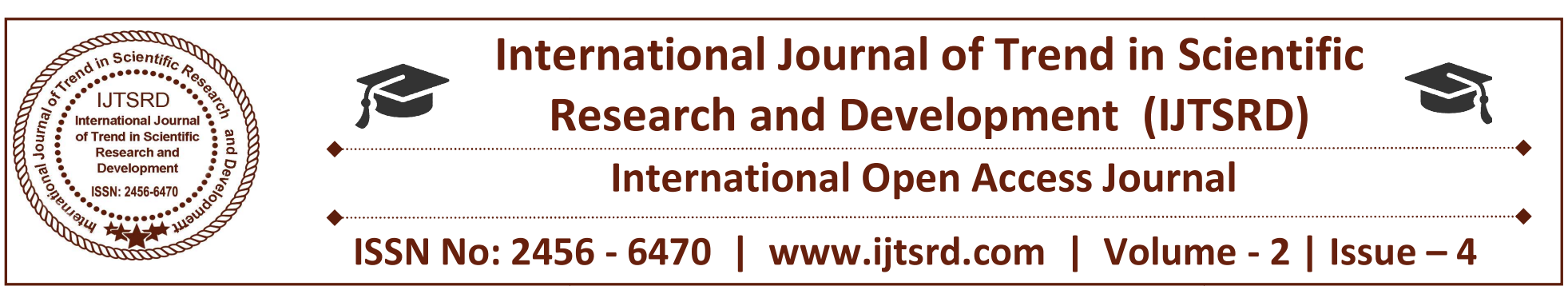

\title{
A Frame Study on Sentiment Analysis of Hindi Language Using Machine Learning Sheetal Sharma ${ }^{1}$, S K Bharti ${ }^{2}$, Raj Kumar Goel ${ }^{2}$ \\ ${ }^{1} \mathrm{M}$. Tech Student, ${ }^{2}$ Assistant Professor \\ Department of Computer Science and Engineering,Noida Institute of \\ Engineering \& Technology, Greater Noida, Uttar Pradesh, India
}

\section{ABSTRACT}

Because of increment in measure of Hindi substance on the web in past years, there are more prerequisites to perform feeling examination for Hindi Language. Conclusion Analysis (SA) is an undertaking which discovers introduction of one's feeling in a snippet of data as for an element. It manages examining feelings, sentiments, and the state of mind of a speaker or an author from a given snippet of data. Estimation Analysis includes catching of client's conduct, different preferences of a person from the content. In this research study HindiSentiWordNet (HSWN) to find the overall sentiment associated with the document; polarity of words in the review are extracted from HSWN and then final aggregated polarity is calculated which can sum as either positive, negative or neutral. Synset replacement algorithm is used to find polarity of those words which don't have polarity associated with it in HSWN. Negation and discourse relations which are mostly present in Hindi movie review are also handled to improve the performance of the system. For this genre we present three different approaches for performing sentiment classification such as-

1. Using Subjective Lexicon

2. N-Gram Method

3. Weighed N-Gram

\section{INTRODUCTION}

Notion Analysis is an errand under common language handling which discovers introduction of a man supposition or emotions over an element [1]. It manages examining individual feelings, sentiments, state of mind and conclusion of a speaker or an author over a question. The essential focus of SA is to discover the assessments communicated by individual over a data or element [2].

Hindi is the fourth most noteworthy talking dialect on the planet. The web contrasted with past years is right now enhanced with non English dialects as well. There exist not very many frameworks which ascertain assumption related with Hindi content as conclusion investigation is exceptionally troublesome for Hindi dialect due various multifaceted nature related with Hindi content. Very much clarified standard corpora are as yet not accessible for Hindi dialect. Hindi dialect needs accessibility of proficient assets like parser and tagger which are basic for separating feeling. HindiSentiWordNet (HSWN) like surely understand English SentiWordNet is accessible yet comprises of constrained quantities of modifiers and verb modifiers, which still needs to change to accomplish higher precision.

The majority of the people groups now a days might want to share their sentiments, encounters and conclusions on the Web. Now individuals usually utilize web journals, gatherings, e-news, audits channels and the social organizing stages, for example, Facebook, Twitter, to express their perspectives and sentiments. The World Wide Web assumes a significant part in get-together general assessment. These assessments are extremely useful for the business associations, as they would think about the conclusions/assessments of their client about their items and furthermore helps the clients in taking the choice. Extensive measure of client content 
information is produced on the Web each day, hence mining the information and recognizing client assumptions, wishes, different preferences is one of an imperative assignment. We present a supervised sentiment/opinion classification model based on the Naive Bayes algorithm.

\section{Goals}

1. To do Sentiment Analysis on Hindi Language, using data mining and machine learning Algorithms.

2. To plot graph between features $\mathrm{v} / \mathrm{s}$ accuracies and find the best accuracy.

There are numerous situations where same words might be utilized as a part of various settings and setting subordinate word mapping is as yet a troublesome assignment. A framework for performing sentiment analysis on Hindi language is presented in this paper. Related works done and past literature is discussed in section 2. Proposed system is defined which uses HSWN to extract polarity associated with sentiment words in section 3. Finally, section 4 concludes the paper.

\section{LITERATURE REVIEW}

Richa Sharma, Shweta Nigam and Rekha Jain (2014) considered on feeling mining or notion. Examination is a characteristic dialect preparing undertaking that mines data from different content structures, for example, surveys, news, and writes and arranges them based on their extremity as positive, negative or impartial.. In this paper a Hindi language opinion mining system is proposed. The system classifies the reviews as positive, negative and neutral for Hindi language. Negation is also handled in the proposed system. Experimental results using reviews of movies show the effectiveness of the system.

Pooja Pandey, SharvariGovilkar (2015) studied on the amount of Hindi content on the web in past years, there are more requirements to perform sentiment analysis for Hindi Language. Sentiment Analysis involves capturing of user's behavior, likes and dislikes of an individual from the text. The work of most of the SA system is to identify the sentiments express over an entity, and then classify it into either positive or negative sentiment. Our proposed system for sentiment analysis of Hindi movie review uses Hindi Senti Word Net (HSWN) to find the overall sentiment associated with the document; polarity of words in the review are extracted from HSWN and then final aggregated polarity is calculated which can sum as either positive, negative or neutral. Synset replacement algorithm is used to find polarity of those words which don't have polarity associated with it in HSWN. Negation and discourse relations which are mostly present in Hindi movie review are also handled to improve the performance of the system.

VandanaJha, Manjunath N, P Deepa Shenoy and Venugopal K R (2016) many works in the area of Sentiment Analysis is available for English language. From last few years, opinion-rich resources are booming in other languages and hence there is a need to perform Sentiment Analysis in those languages. In this paper, a Sentiment Analysis in Hindi Language (SAHL) is proposed for reviews in movie domain. We have used Naive Bayes Classifier, Support Vector Machine and Maximum Entropy techniques for Machine learning. In Lexicon based classification, adjectives are considered as opinion words and according to the polarity of the adjectives, the documents are classified, negation handling with window size consideration for improving the accuracy of classification. The effectiveness of the proposed approach is confirmed by extensive simulations performed on a large movie dataset.

\section{Data Collection For Hindi Language}

Tonextract sentiment associated with Hindi documents, HindiSentiWordNet (HSWN) will be used which consists of Hindi sentiment words and their associated positive and negative polarity. Here existing HSWN is improved by adding missing sentimental words related to Hindi movie domain. First a Hindi movie review dataset will be created, as mostly the data for finding sentiment is usually present on web and bears lot of noises like numbers, one length terms and all which mostly don't contribute in further processing to find sentiment polarity of the input therefore such data will be thoroughly processed to remove unwanted data in the dataset

Our proposed approach performs Sentiment Analysis of Hindi documents using HindiSentiWordNet (HSWN). During the first stage we are improving the existing HSWN with the help of English SentiWordNet, where sentimental words which are not present in the HSWN are translated to English and then searched in English SentiWordNet to retrieve their polarity. In the second stage, sentiment is extracted by finding the overall polarity of the 
document; which can be positive, negative or neutral. the document. Finally, overall sentiment orientation of Here during pre-processing tokens are extracted from the document is determined by aggregating the sentence and spell check is performed. Rules are polarity values of all the sentimental words in the devised for handling negation and discourse relation which highly influence the sentiments expressed in document.

\subsection{System Improvisation}

To perform sentiment analysis in Indian language, the data set has to be prepared first. To prepare the data set, large numbers of Hindi news sentences were collected from the Web. There are lots of websites like which contain Hindi content. Here, Hindi news sentences were collected from the Hindi newspapers website. But before applying as an input, the collected data first preprocessed. After preprocessing the reviews were applied as an input. All the data after getting preprocessed, it is taken as input for the analysis system and using algorithms, we get the results in the form of output as shown in Figure 1.

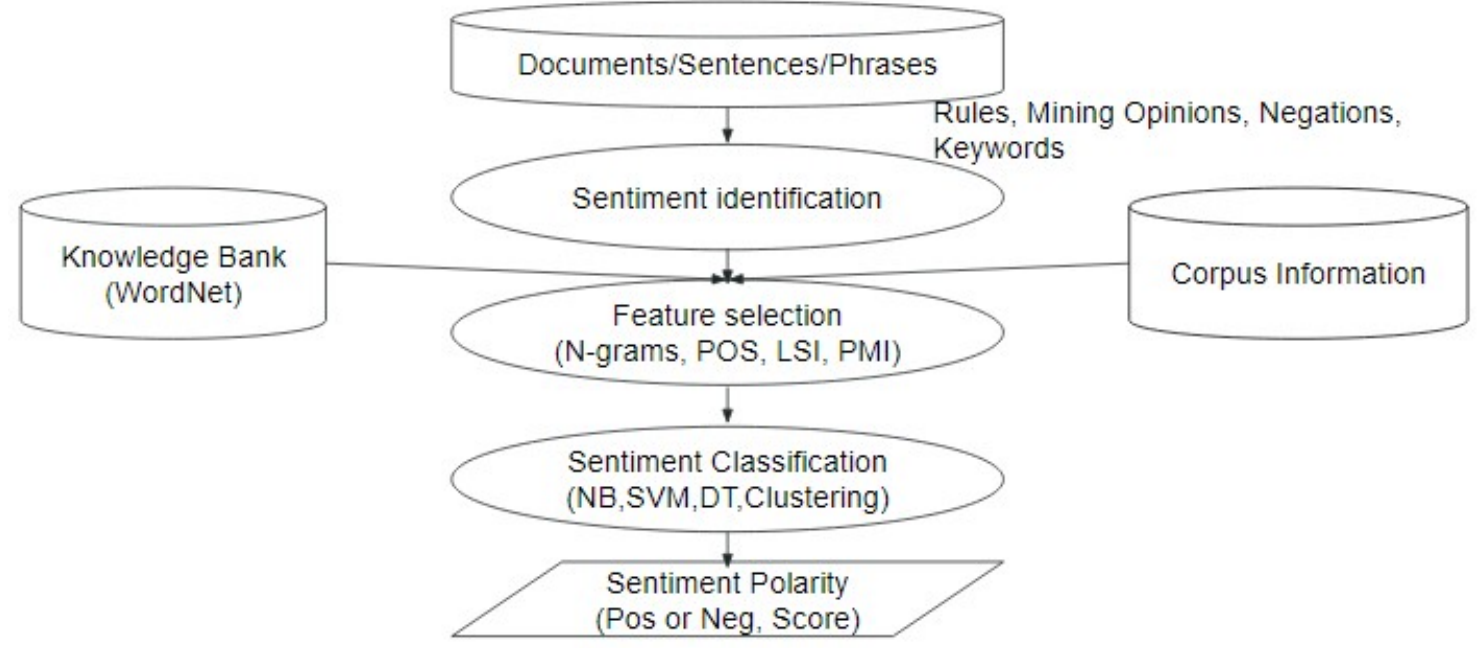

Figure 3.1: Sentiment Analysis Process

\subsection{Improving Hindi Senti Word Net}

In this phase existing version of HindiSentiWordNet is improved, as there are limited numbers of adjectives and adverbs i.e. sentiment bearing words present in the existing HSWN so there is need of adding all those missing sentiment bearing words to get more accurate results. HSWN is created using the Hindi WordNet and English SentiWordNet (SWN) by matching the words having same synset IDs. While HSWN was created for Hindi language, it was considered that all synonyms of a particular sentimental word have the same polarity while all antonyms would have the reverse polarity of that word. 


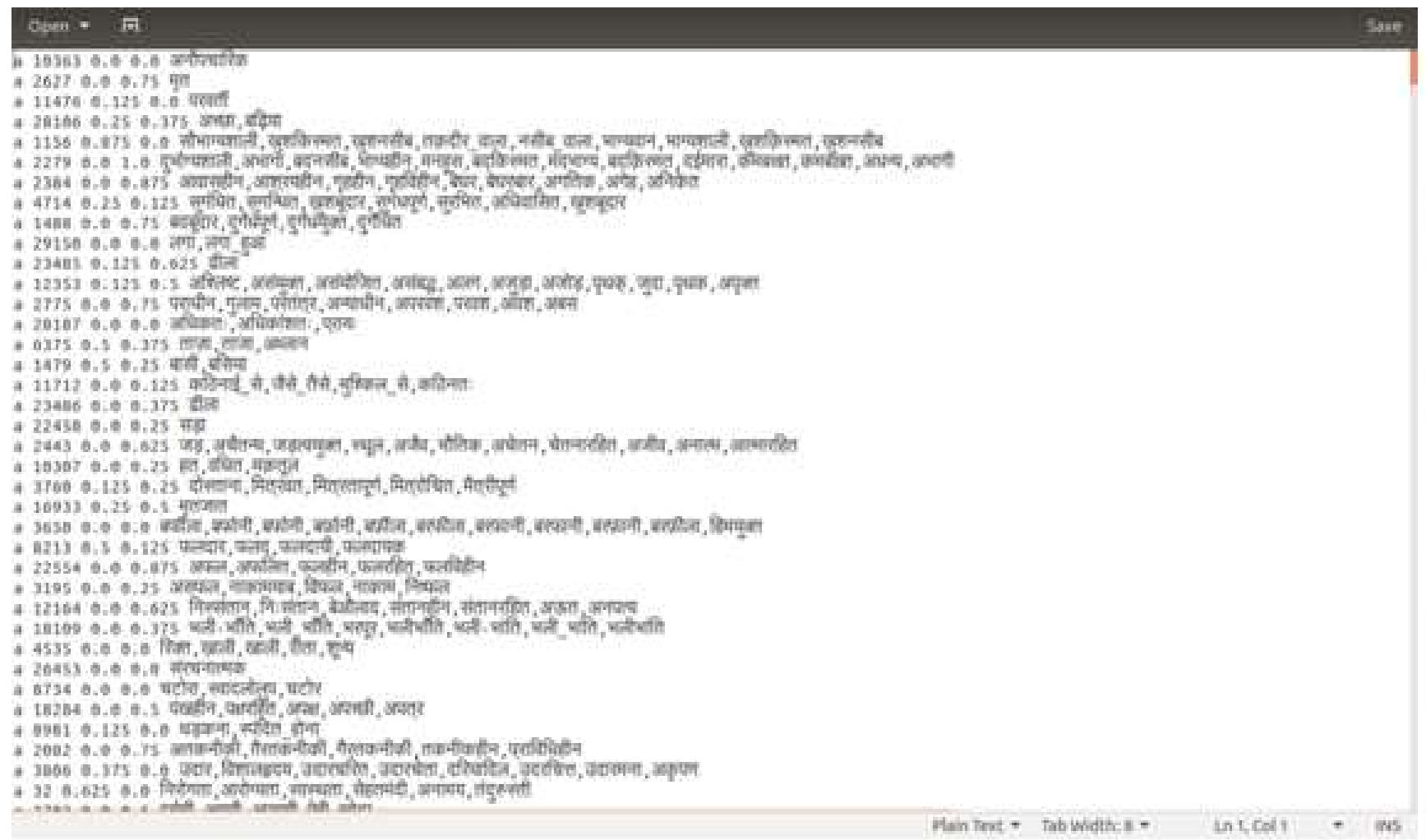

Figure 3.2: Sentiment Analysis Process

\subsection{Naïve Bayes Classifier}

The Naive Bayes model involves a simplifying conditional independence assumption. That is given a class (positive or negative), the words are conditionally independent of each other. This assumption does not affect the accuracy in text classification by much but makes really fast classification algorithms applicable for the problem. In our case, the maximum likelihood probability of a word belonging to a particular class is given by the expression:

\subsection{Data Preprocessing}

$$
P\left(x_{i} \mid c\right)=\frac{\text { Count of } x_{i} \text { in documents of class } c}{\text { Total no of words in documents of class } c}
$$

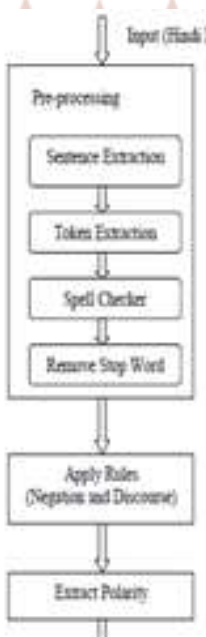

Figure 3.3: Sentiment Analysis Process 


\section{RESULTS AND DISCUSSION}

In the Hindi sentiments orientation systems is performed on the Hindi news sentences domain. The experiments have been performed by using of Hindi news sentences domain. Table 2 presents the data of Hindi sentiments in terms of sentences, train positive and negative as input data.

Table 2: Input Data

\begin{tabular}{|l|l|}
\hline Measure & Results \\
\hline Positive Sentences & 1400 \\
\hline Negative Sentences & 1500 \\
\hline Train Positive & 1333 \\
\hline Train Negative & 1352 \\
\hline Test positive & 149 \\
\hline Test Negative & 149 \\
\hline
\end{tabular}

Table3: output data

\begin{tabular}{|l|l|}
\hline Measure & Results \\
\hline $\begin{array}{l}\text { Total no of times positive } \\
\text { word occurred in data set }\end{array}$ & 15567 \\
\hline $\begin{array}{l}\text { Total no of times negative } \\
\text { word occurred in data set }\end{array}$ & 17480 \\
\hline Total no of features & 2839 \\
\hline optimal value of $\mathrm{k}$ & 2200 \\
\hline Best accuracy & 0.689641994751 \\
\hline
\end{tabular}

Figure 4.1: Graphical representation of results in the form of polarity of Hindi words

\section{CONCLUIOSN}

Sentiment Analysis using Data Mining is an emerging research field and is very important because human beings are largely dependent on the web nowadays.In this paper an overview of the Hindi based Sentiment Analysis in Hindi Language using Data Mining has given, based on existing researches that has been performed in Hindi language. Techniques and several challenges of Hindi based sentiment analysis are also discussed. But performing sentiment analysis in Hindi language is not an easy task, because the nature of
Indian languages varies a great deal in terms of the script, representation level and linguistic characteristics, etc. To understand the behaviour of Indian languages, large amount of work needs to be done in the field of opinion mining for Hindi language. The best $\mathrm{k}$ was found at instance 2200 and accuracy was found about 0.689641994751 at the given dataset (3000 Hindi News Sentences from hindi new wesites.).

\section{REFERENCES}

1. Richa Sharma,Shweta Nigam and Rekha Jain (2014) "Polarity Detection Of Movie Reviews in Hindi Language" International Journal on Computational Sciences \& Applications (IJCSA) Vol.4, No.4, August 2014.

2. Pooja Pandey, Sharvari Govilkar (2015) "A Framework for Sentiment Analysis in Hindi using HSWN" International Journal of Computer Applications, vol. 119, 23-26.

3. Vandana Jha, Manjunath N, P Deepa Shenoy and Venugopal K R (2016) "Sentiment Analysis in a Resource Scarce Language:Hindi" International Journal of Scientific \& Engineering Research, Volume 7, Issue 9, pp no 968-990.

4. Namita Mittal and Basant Agarwal (2013) "Sentiment Analysis of Hindi Review based on Negation and Discourse Relation" International Joint Conference on Natural Language Processing, pages 45-50, Nagoya, Japan, 14-18 October.

5. Shanta Phani, Shibamouli Lahiri and Arindam Biswas (2016) "Sentiment Analysis of Tweets in Three Indian Languages" Proceedings of the 6th Workshop on South and Southeast Asian Natural Language Processing, pages 93-102, Osaka, Japan, December.

6. Richa Sharma1, Shweta Nigam2 and Rekha Jain (2014b) "Opinion Mining In Hindi Language: A Survey" International Journal in Foundations of Computer Science \& Technology (IJFCST), Vol.4, No.2, March 2014.

7. Komal Garg and Preetpal Kaur Buttar (2015) "Aspect based Sentiment Analysis of Hindi Text Review" International Journal of Advanced Research in Computer Science, Volume 8, No. 7, July - August 2017

8. Namita Mittal, Basant Agarwal, Garvit Chouhan, Prateek Pareek, and Nitin Bania (2013) "Discourse Based Sentiment Analysis for Hindi Reviews" P. Maji et al. (Eds.): PReMI 2013, LNCS 8251, pp. 720-725, 2013. 